\title{
A Modified SweepSAR Mode with Dual Channels for High Resolution and Wide Swath
}

\author{
Seong Sik Yoon ${ }^{1} \cdot$ Jae Wook Lee ${ }^{1, *} \cdot$ Taek-Kyung Lee ${ }^{1} \cdot$ Sang-burm Ryu ${ }^{2}$. \\ Hyeon-Cheol Lee ${ }^{2} \cdot$ Sang Gyu Lee ${ }^{2}$
}

\begin{abstract}
In this study, an imaging mode of the modified SweepSAR is proposed with performance analysis for a high-resolution and wide swath coverage. To reduce the overall antenna size required for the solution of the azimuth ambiguity problem, different pulse repetition frequencies (PRFs) are utilized for different transmitters, respectively. For each imaging mode, system performance parameters are used for simulation, analysis, wide swath prediction, and comparison between conventional ScanSAR mode and SweepSAR mode based on scanon-receive (SCORE). The system parameters of AASR, RASR, and NESZ will be estimated and suggested on the imaging mode by using appropriate reflector antenna with the effectiveness of a modified SweepSAR employing dual channels.
\end{abstract}

Key Words: Ambiguity, Antenna Pattern, High Resolution, NESZ, PRF, SweepSAR, Wide Swath.

\section{INTRODUCTION}

Recent developments in radar imaging technology require high resolution and wide swath for accurate target detection within allowable satellite revisit times. High-resolution target discrimination can potentially be enhanced by multi-band, multi-polarization-mode [1], and multi-beam technologies [2]. Antenna systems and transmit-receive modules are also considered and examined as prospective components for the development of a highly effective synthetic aperture radar (SAR) payload given that their compactness and light weight can contribute to the reduction of overall payload weight.

Observation coverage can be expanded through several techniques, including the application of low carrier signals, the reduction of an antenna's physical length, and the high-altitude operation of a satellite platform, taking into account the low
SNR characteristics. ScanSAR mode operates under an illuminating antenna beam projected in the elevation direction and sequentially receives burst signals [3]. A drawback of this imaging mode, however, is that the dwell time of beams that illuminate terrain becomes shorter than that achieved in stripmap mode because of the use of echo signals from only a part of an observation region. Accordingly, the Doppler resolution increases proportionally to the number of sub-swaths [4]. To achieve high-resolution and wide-swath SAR imaging, researchers suggested the use of a displaced phase center antenna (DPCA) and an extension of the DPCA called a quad-element rectangular array, along with multiple sub-aperture elements in receiving antenna systems. As a basic principle of the DPCA, samples of data collected along the flight direction are required for pulse transmission performed through the combination of a multichannel receiver and corresponding multiple aperture ele-

Manuscript received February 8, 2018 ; Revised May 4, 2018 ; Accepted June 4, 2018. (ID No. 20180208-016J)

${ }^{1}$ Department of Electronics and Information Engineering, Korea Aerospace University, Goyang, Korea.

${ }^{2}$ Payload Electronics Team, Korea Aerospace Research Institute, Daejeon, Korea.

"Corresponding Author: Jae Wook Lee (e-mail: jwlee1@kau.ac.kr)

This is an Open-Access article distributed under the terms of the Creative Commons Attribution Non-Commercial License (http://creativecommons.org/licenses/by-nc/4.0) which permits unrestricted non-commercial use, distribution, and reproduction in any medium, provided the original work is properly cited.

(c) Copyright The Korean Institute of Electromagnetic Engineering and Science. All Rights Reserved. 
ments, which suppress azimuth ambiguities by using displaced phase centers and/or multiple azimuth beams $[5,6]$. As another approach for the improvement of the ambiguity problem, the Terrain Observation Progress Scan SAR (TOPSAR), which works as a ScanSAR mode with the characteristics of reducing the scalloping effects by including additional scanning in the azimuthal direction. Its disadvantages, however, are that it requires $2 \mathrm{D}$ phased array antenna systems and involves complexity in the design of transmitter/receiver modules [7]. In contrast to conventional and newly proposed high-resolution and wideswath approaches, SweepSAR improves azimuth resolution; it requires transmitters to use a wide beamwidth for wide coverage and requires receivers to sweep the echo signals reflected from each narrow region divided into several sub-swaths [8]. In this mode, a low pulse repetition frequency (PRF) and a long antenna are necessary to transmit a single beam and compensate for the performance degradation stemming from azimuth ambiguities [9].

In this paper, we present a new SAR imaging mode that transmits two signals that correspond to PRFs to overcome the ambiguity problem caused by low $\mathrm{PRF}$ - a solution that is achieved even under a reduced antenna size. Section II gives a detailed explanation of the imaging mode proposed in this study with the main system parameters. Section III presents the comparison results according to the various parameters.

\section{DESIGN OF MODIFIED SWEEPSAR}

\section{System Overview}

The azimuthal resolution of ScanSAR mode proportionally increases with the number of sub-swaths. Conversely, in SweepSAR mode, multiple signals are transmitted through a feed array with a feedhorn in an elevation direction over a wide coverage, and reflected echo signals are sequentially received with a precise calculation of timing interval. These features result in high resolution and wide swath. At this stage, a selected PRF should be sufficiently low to prevent the timing of a received signal from overlapping with the timing of a signal transmitted in an overall swath area. Generally, as PRFs decrease/increase, range/azimuth ambiguities decrease. In addition, the inclusion of a wide Doppler bandwidth increases the azimuth ambiguity-to-signal ratio (AASR). Specifically, in the azimuth direction, a SAR system transmits pulses periodically in a given PRF, which represents the return signals collected within the range of the Nyquist sampling requirement. Therefore, antenna length should be larger than twice the satellite speed divided by the PRF, as indicated in [9]. A long antenna length increases the total weight and launching cost of payload.

To overcome the disadvantages of resolution degradation due to increased antenna length (i.e., Azimuth resolution $\left(\rho_{A}\right)$ is

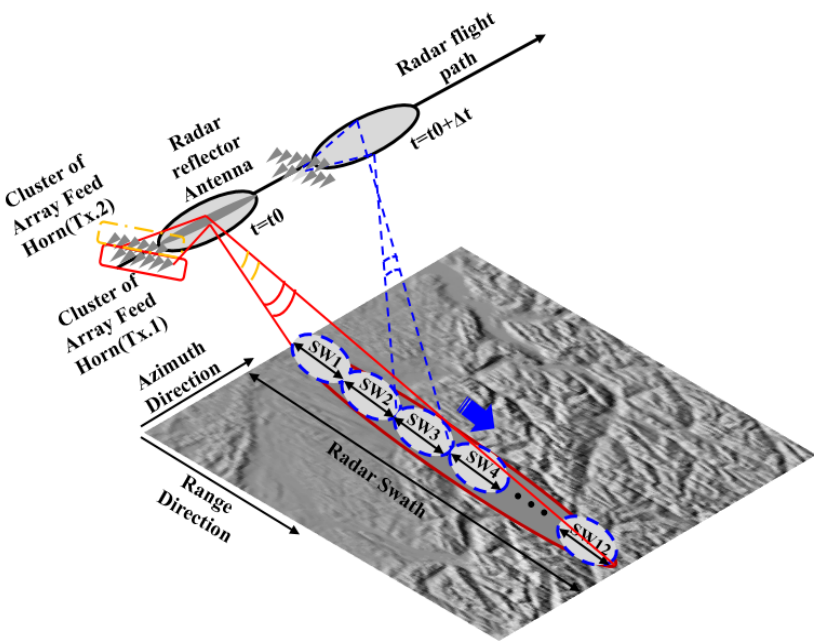

Fig. 1. Geometrical structure of the proposed M-SweepSAR mode.

proportional to antenna length; for example, $\rho_{A}=L / 2$ ) and the asymmetric beam patterns caused by offset feeding structures, we developed a modified SweepSAR (M-SweepSAR) system with a new imaging scenario. Fig. 1 shows the geometrical structure of M-SweepSAR. In this system, the frequency used is assumed to be the C-band, which is highly applicable to disaster/water-level monitoring, and the number of sub-swaths is assumed to be 12 . Table 1 summarizes the main parameters used in the performance evaluation of the proposed SAR system. The total radar swath range is $150 \mathrm{~km}$, and the reflected signals sequentially come from a near range in the order of array. As shown in Fig. 2, two PRF signals higher than $2,500 \mathrm{~Hz}$ are simultaneously transmitted into the overall swath area to avoid a blind range, which is the area of overlap between the timing of a transmitted signal and the timing of a signal returned from the nadir flight direction. With regard to the receipt of echo signals, the signals reflected from sub-swaths 1-7 are sequentially received through the feedhorn of channel 1 , and the signals returned from sub-swaths 8-12 are received through channel 2 . Two rows of feedhorn arrays are aligned in the azimuth direc-

Table 1. Simulation parameters for satellite SAR payload

\begin{tabular}{lc}
\multicolumn{1}{c}{ Parameter } & Value \\
\hline Satellite mean altitude $(\mathrm{km})$ & 505 \\
Earth radius $(\mathrm{km})$ & 6,378 \\
Satellite velocity $(\mathrm{m} / \mathrm{s})$ & 7,610 \\
Total loss $(\mathrm{dB})$ & 2.3 \\
System noise temperature $(\mathrm{K})$ & 290 \\
Target swath width $(\mathrm{km})$ & 150 \\
Incidence angle $\left(^{\circ}\right)$ & $20-35$ \\
RF center frequency $(\mathrm{GHz})$ & 5.4 \\
RF peak power $(\mathrm{W})$ & 2,400 \\
Pulse length $(\mu \mathrm{s})$ & 30 \\
Noise figure $(\mathrm{dB})$ & 6 \\
\hline
\end{tabular}




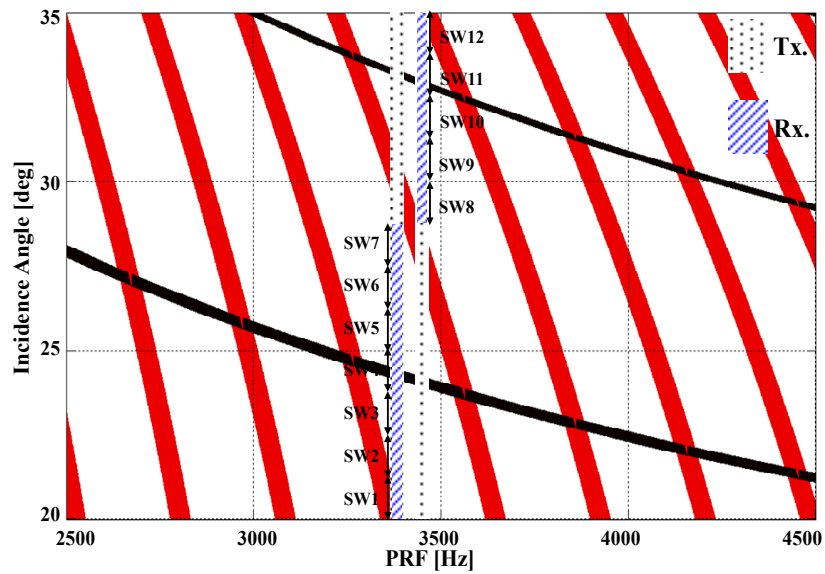

Fig. 2. Timing diagram of the proposed M-SweepSAR mode.

tion for transmitting and receiving signals from the two channels, and appropriate RF up/down converters corresponding to the assumed carrier frequencies are assigned.

\section{SAR System Parameters}

Obtaining a high-quality image necessitates the use of highquality raw image data before commencing with advanced image signal processing. Deriving reasonable results requires an optimized pulse shape and appropriate antenna patterns. An optimized PRF must also be determined by considering the timing sequences of transmitter signals and interference to the receipt of timing sequences from the nadir direction. The performance of an SAR system is rigorously analyzed by determining the main parameters for the imaging mode suitable for a given mission and optimized antenna patterns and then estimating and evaluating these parameters; such parameters include the ambiguity ratio and noise equivalent sigma zero (NESZ) $[10,11]$. Parameter determination should include the impulse response for a point target through signal processing. For example, applying fast Fourier transform to raw data enables the estimation of impulse response characteristics along/ cross a track distance on the ground with sinc-like function behaviors. The demodulated baseband signal, $s_{0}(t, \eta)$, which is received from a point target is represented by [3],

$$
\begin{aligned}
s_{0}(t, \eta)= & A_{0} \omega_{r}[t-2 R(\eta) / c] \omega_{a}\left[\eta-\eta_{c}\right] \\
& \times \exp \left[-j 4 \pi f_{0} R(\eta) / c\right] \exp \left[j \pi K_{r}(t-2 R(\eta) / c)^{2}\right],
\end{aligned}
$$

where $t$ is the range fast time, $\eta$ is the azimuth slow time, $\eta_{c}$ denotes the beam center crossing time, $A_{0}$ represents an arbitrary complex constant, $\omega_{r}(t)$ is the pulse envelope in range, and $\omega_{a}(\eta)$ denotes the magnitude of the azimuth signal. For general considerations, all signals examined within a processing beamwidth are assumed to be coherent with one another, and ScanSAR mode uses a number of $\mathrm{N}$ sub-swaths that correspond to a sin- gle burst duration. If a "gap" between burst trains occurs, zeros are inserted into intervals (zero padding) using a full-aperture algorithm [12].

\section{PERFormance of Modified SwEEPSAR}

This section discusses the comparison of the electrical performance of three imaging modes, namely, ScanSAR mode; the SweepSAR mode for high-precision, wide-area observation; and M-SweepSAR mode.

\section{Antenna Patterns}

The reflector antenna adopted in this work is based on array feedhorns that are arranged in two rows; this arrangement results in feed blockage effects, in contrast to the case of a singlearrayed feeding structure. To avoid blockage effects, we suggest using the offset reflector antenna shown in Fig. 3, which has a parent reflector $\left(\mathrm{D}_{\mathrm{p}}\right)$-to-real reflector $(\mathrm{D})$ diametrical ratio of 0.45 and a real reflector (D)-to-focal length $\left(F_{p}\right)$ ratio of 0.9 . The antenna lengths $(\mathrm{L})$ in the azimuth direction were set to 3, 13.5, and $6 \mathrm{~m}$ for ScanSAR, SweepSAR, and M-SweepSAR, respectively, with appropriate PRFs. Depending on imaging mode, the number of sub-swaths was established in such a way as to cover overall wide swath. The number of sub-swaths in ScanSAR is 6, and the selected PRF for avoiding a blind range is 6,200-7,300 Hz. The numbers of sub-swaths for SweepSAR and M-SweepSAR are 24 and 12 , respectively, at $1,710 \mathrm{~Hz}$, and their PRFs are 3,390 and 3,450 Hz, respectively. The distance $\left(\delta_{\mathrm{F}}\right)$ between the two nearest feeding elements was deter-

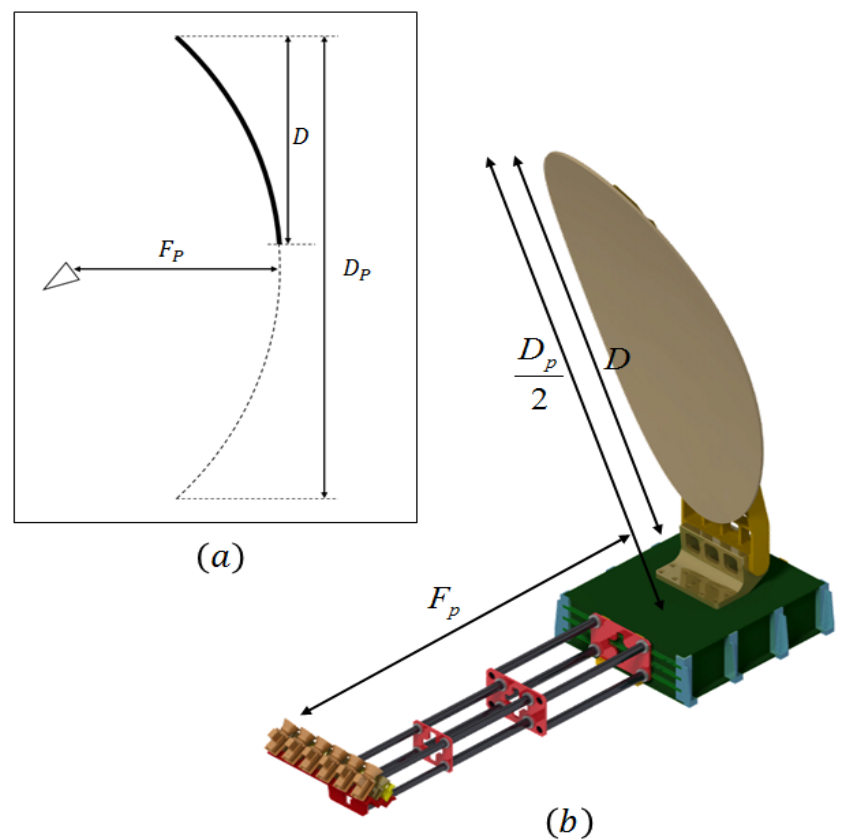

Fig. 3. Offset reflector antenna with array feed for satellite SAR performance analysis. (a) Side view, (b) perspective view. 
mined to avoid grating lobe generation in the beam patterns from the feedhorn located within the physical range of the real reflector:

$$
\delta_{F}<\frac{\lambda}{\cos \left(\left(\theta_{\text {edge }}-\pi\right) / 2\right)}
$$

Figs. 4-9 show the radiation patterns in each imaging mode; the patterns were generated with reflector antennas through the use of a commercially available simulation tool (GRASP) based on physical optics. As illustrated in Figs. 5 and 6, the gain difference from the zero angle to both end points is due to the phase differences between center feeding and end feeding in the array feeding system.

\section{Comparison of the Performance of Imaging Modes}

On the basis of the evaluation formulas for system performance and the radiation patterns of three antennas that were assumed to be present based on the three imaging modes, the

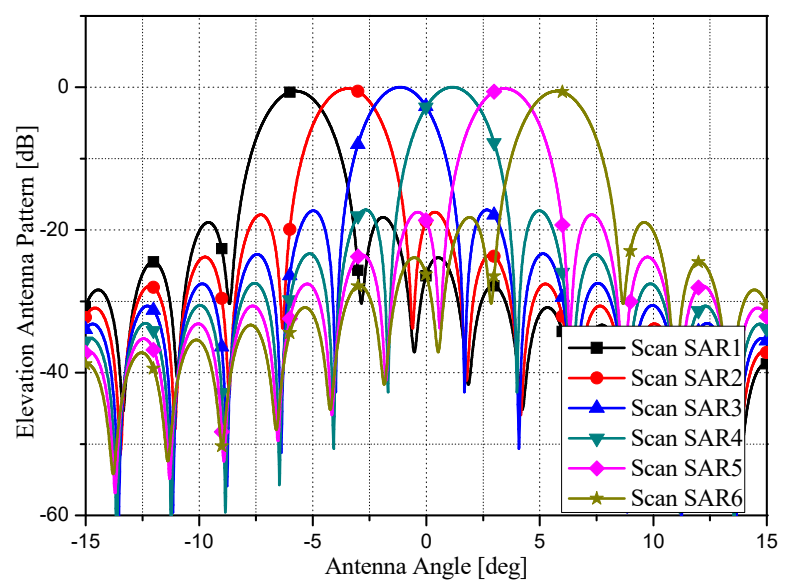

Fig. 4. Elevation radiation patterns of the offset reflector antenna for ScanSAR.

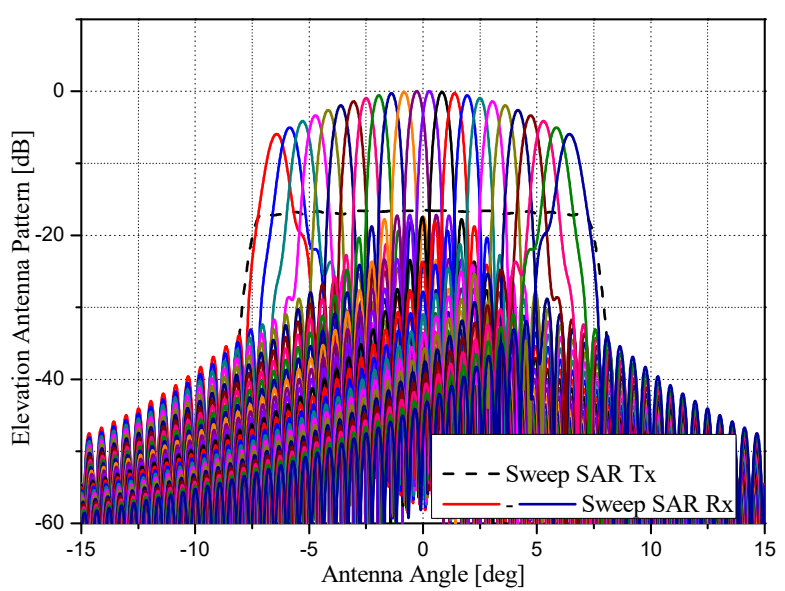

Fig. 5. Elevation radiation patterns of the offset reflector antenna for SweepSAR.

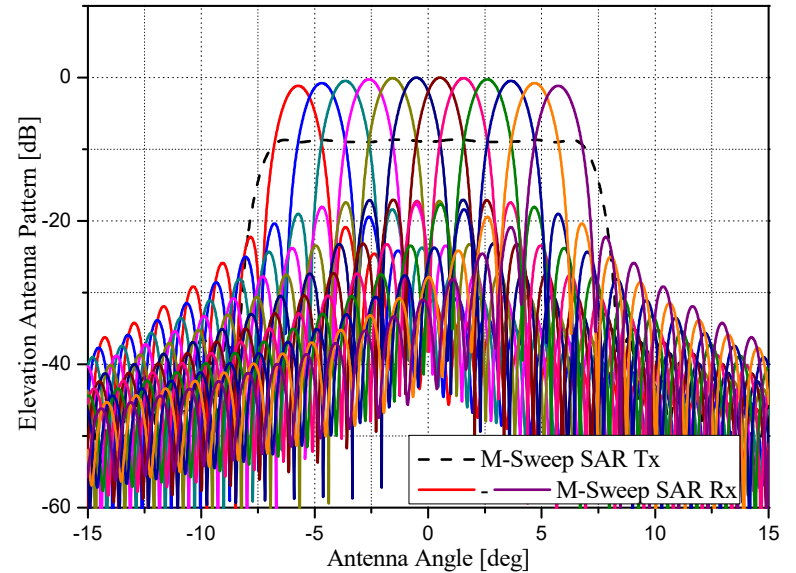

Fig. 6. Elevation radiation patterns of the offset reflector antenna for M-SweepSAR.

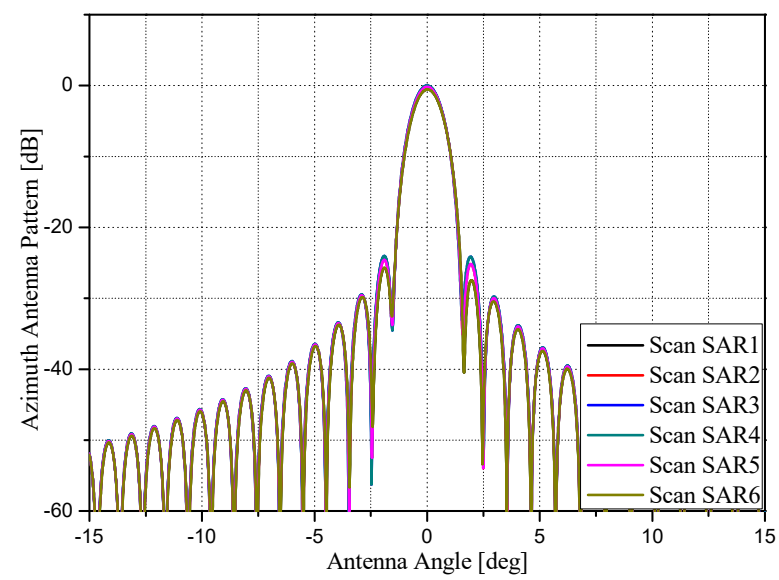

Fig. 7. Azimuth radiation patterns of the offset reflector antenna for ScanSAR.

range ambiguity-to-signal ratio (RASR), AASR, NESZ, and azimuth impulse responses related to resolution were examined by applying the surface reflectivity model [13] on the ground surface with reference to the incidence angle. In the NESZ es-

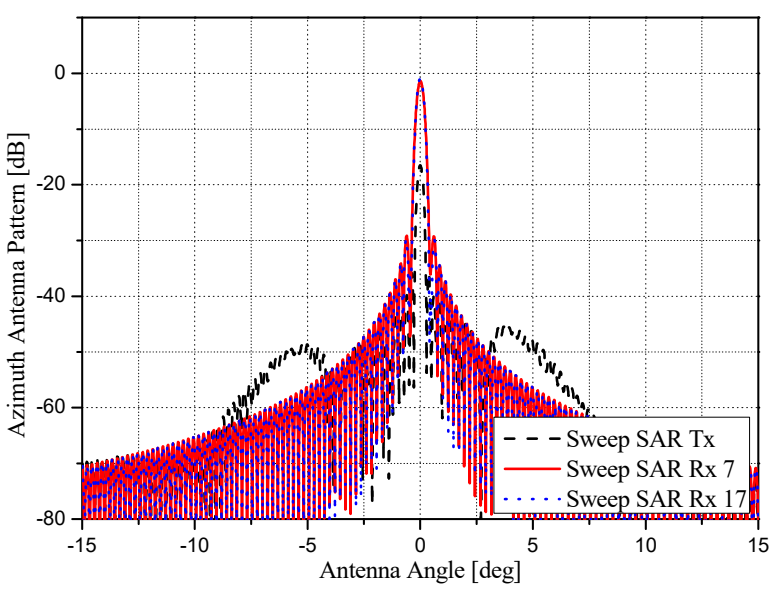

Fig. 8. Azimuth radiation patterns of the offset reflector antenna for SweepSAR. 


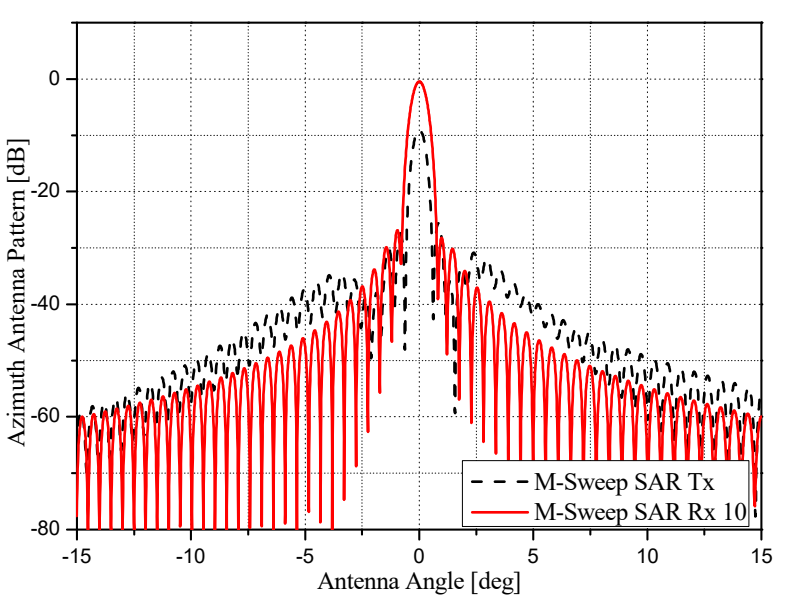

Fig. 9. Azimuth radiation patterns of the offset reflector antenna for M-SweepSAR.

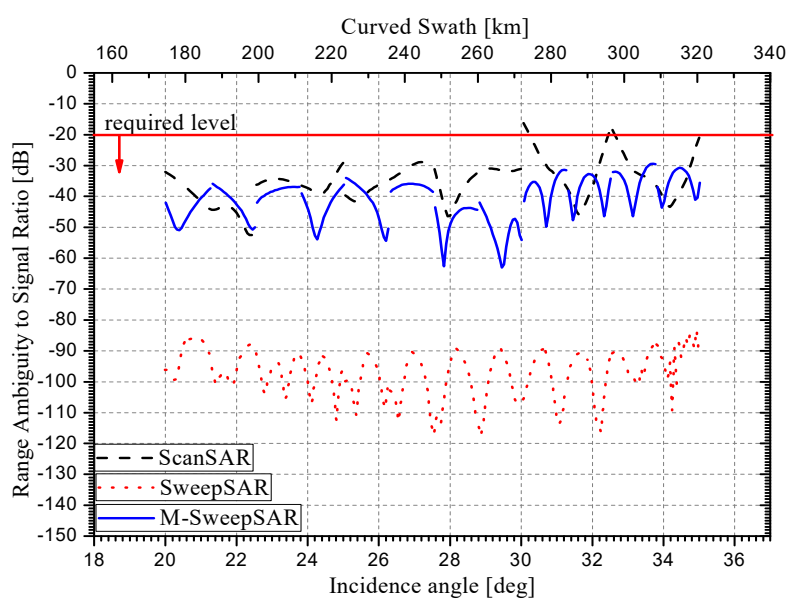

Fig. 10. Range ambiguity-to-signal ratio depending on imaging mode.

timation, we assumed peak power as an input power having a constant level of $2.4 \mathrm{~kW}$ in all the three imaging modes. Figs. 10-12 illustrate the SAR performance of each imaging mode. SweepSAR is the best imaging mode in terms of RASR because it has the lowest PRF selection (Fig. 10), whereas MSweepSAR exhibits the best performance mode with respect to AASR in the vicinity of the relatively far range because of its high PRF (Fig. 11). All the imaging modes satisfy the requirement of NESZ levels under $-20 \mathrm{~dB}$, but SweepSAR mode shows the best performance in this regard because of its high gain antenna. Nevertheless, the flatness of SweepSAR mode's AASR and NESZ (Figs. 11 and 12) is poor because of the phase difference from both ends of the radiation patterns in the elevation direction. As a final check on SAR system performance, we probed into azimuth impulse responses related to resolution in each of the imaging modes. Assuming a uniform gain in the range of a 3-dB beamwidth from the reflector antenna within the processing bandwidth, we obtained a peak sidelobe ratio of about $-13.4 \mathrm{~dB}$. The resolutions of ScanSAR,

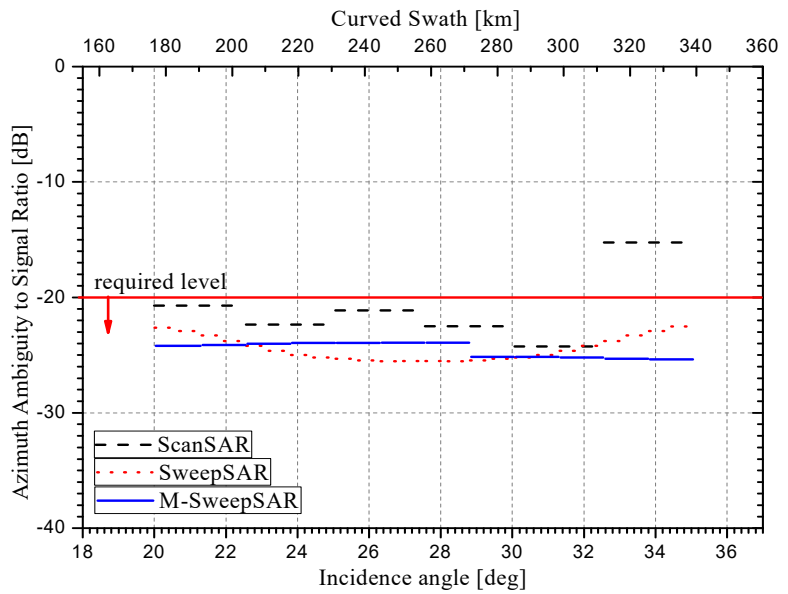

Fig. 11. Azimuth ambiguity-to-signal ratio depending on imaging mode.

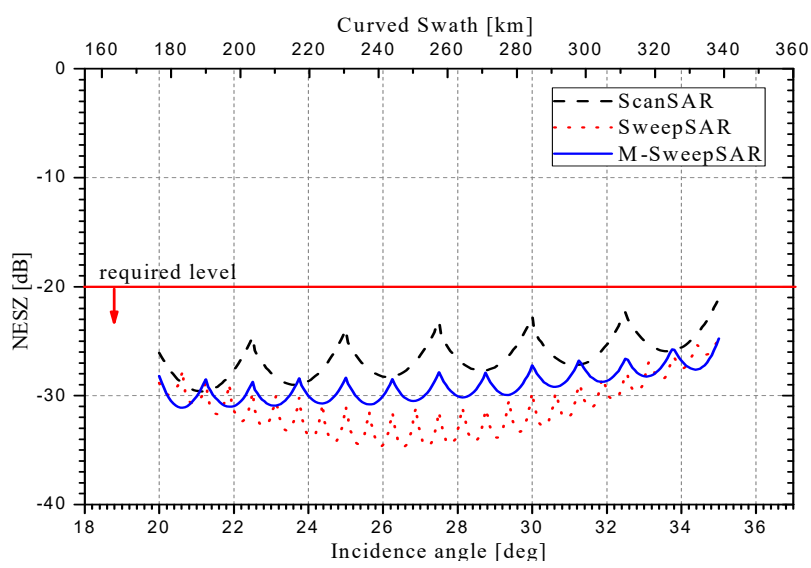

Fig. 12. Noise equivalent sigma zero depending on imaging mode.

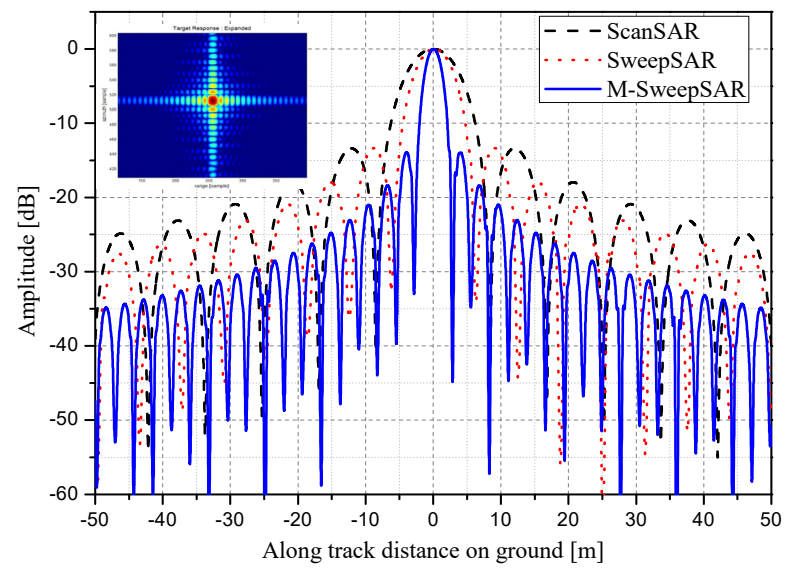

Fig. 13. Azimuth impulse responses depending on imaging mode.

SweepSAR, and M-SweepSAR are 7.56, 5.25, and $2.45 \mathrm{~m}$ (Fig. $13)$, indicating that M-SweepSAR is the best imaging mode in terms of resolution.

\section{CONCLUSION}

An effective M-SweepSAR system was developed to reduce 
antenna size to a level suitable for high-resolution, wide-swath coverage. The performance of the proposed system was analyzed and compared with that of ScanSAR and SweepSAR. An appropriate antenna design and a suitable PRF were selected for each of the imaging modes, and system parameter formulas were applied to estimate the AASR, RASR, and NESZ values of the compared modes. Additionally, azimuth impulse responses were predicted for the maximum azimuthal resolution from raw data generated from a target point. For the same observation swath area, M-SweepSAR exhibits reasonable, good, and meaningful performance, with an antenna reduced to more than half of a SweepSAR antenna. The proposed analysis technique is also expected to be easily implementable to appropriate system designs and imaging modes. As an important factor, the isolation between transmitters and receivers should be further investigated given that spaceborne systems require a high power transmitter.

This research was performed for the Development of Radar Payload Technologies for Compact Satellite in Korea Aerospace Research Institute, funded by the Ministry of Science and ICT.

\section{REFERENCES}

[1] V. Turkar, R. Deo, Y. S. Rao, S. Mohan, and A. Das, "Classification accuracy of multi-frequency and multipolarization SAR images for various land covers," IEEE Journal of Selected Topics in Applied Earth Observations and Remote Sensing, vol. 5, no. 3, pp. 936-941, 2012.

[2] A. Currie and M. A. Brown, "Wide-swath SAR," IEE Proceedings F (Radar and Signal Processing), vol. 139, no. 2, pp. 122-135, 1992.

[3] I. G. Cumming and F. H. Wong, Digital Processing of Synthetic Aperture Radar Data Algorithm and Implementation. Boston, MA: Artech House, 2005.

[4] D. D'Aria, F. D. Zan, D. Giudici, A. M. Guarnieri, and F. Rocca, "Burst-mode SARs for wide-swath surveys," Canadian Journal of Remote Sensing, vol. 33, no. 1, pp. 27-38,
2007.

[5] G. Krieger, N. Gebert, and A. Moreira, "Unambiguous SAR signal reconstruction from nonuniform displaced phase center sampling," IEEE Geoscience and Remote Sensing Letters, vol. 1, no. 4, pp. 260-264, 2004.

[6] G. D. Callaghan and I. D. Longstaff, "Wide-swath spaceborne SAR using a quad-element array," IEE ProceedingsRadar, Sonar and Navigation, vol. 146, no. 3, pp. 159-165, 1999.

[7] F. De Zan and A. M. Guarnieri, "TOPSAR: terrain observation by progressive scans," IEEE Transactions on Geoscience and Remote Sensing, vol. 44, no. 9, pp. 2352-2360, 2006.

[8] M. Younis, S. Huber, A. Patyuchenko, F. Bordoni, and G. Krieger, "Performance comparison of reflector-and planarantenna based digital beam-forming SAR," International Journal of Antennas and Propagation, vol. 2009, article ID. 614931, 2009.

[9] G. Krieger, N. Gebert, M. Younis, F. Bordoni, A. Patyuchenko, and A. Moreira, "Advanced concepts for ultrawide-swath SAR imaging," in Proceedings of 2008 7th European Conference on Synthetic Aperture Radar (EUSAR), Friedrichshafen, Germany, 2008, pp. 1-4.

[10] S. S. Yoon, J. W. Lee, T. K. Lee, and D. W. Yi, "Parameter selection procedure of parabolic reflector antenna for the optimum synthetic aperture radar performances," Journal of Electromagnetic Engineering and Science, vol. 13, no. 4, pp. 251-258, 2013.

[11] S. Y. Kim, N. H. Myung, and M. J. Kang, "Antenna mask design for SAR performance optimization," IEEE Geoscience and Remote Sensing Letters, vol. 6, no. 3, pp. 443-447, 2009.

[12] R. Bamler and M. Eineder, "ScanSAR processing using standard high precision SAR algorithms," IEEE Transactions on Geoscience and Remote Sensing, vol. 34, no. 1, pp. 212-218, 1996.

[13] M. H. Ka and A. A. Kononov, "Effect of look angle on the accuracy performance of fixed-baseline interferometric SAR," IEEE Geoscience and Remote Sensing Letters, vol. 4, no. 1, pp. 65-69, 2007. 


\section{Seong Sik Yoon}

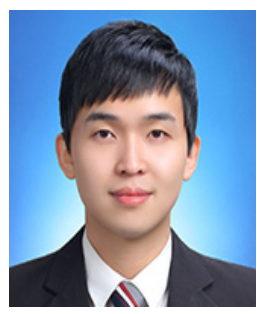

received his B.S., M.S., and Ph.D. degrees in electronic engineering from Korea Aerospace University in Goyang, Korea in 2010, 2013, and 2018, respectively. He is currently senior researcher at Laboratory of Radar PGM in Hanwha Systems, Yongin, Korea. His research interests include satellite communication antennas, radar antenna design and analysis, and spaceborne SAR system analysis.

\section{Jae Wook Lee}

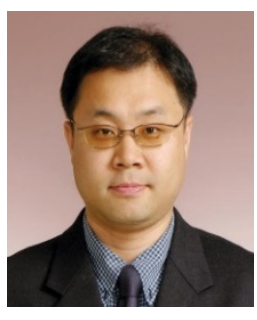

received his B.S. degree in electronic engineering from Hanyang University in Seoul, Korea in 1992 and his M.S. and Ph.D. degrees in electrical engineering (major in electromagnetics) from the Korea Advanced Institute of Science and Technology (KAIST), Daejeon, Korea in 1994 and 1998, respectively. From 1998 to 2004, he served as a senior member of the Advanced Radio Technology Department, Radio and Broadcasting Research Laboratory, and Electronics and Telecommunications Research Institute in Daejeon, Korea. He then joined the faculty of Korea Aerospace University in Goyang, Korea, where he is currently a professor in the School of Electronics and Information Engineering. His research interests include high power amplifier design, computational electromagnetics, electromagnetic interference or electromagnetic compatibility analysis on printed circuit boards, electromagnetic pulse analysis, satellite antennas, and spaceborne SAR systems.

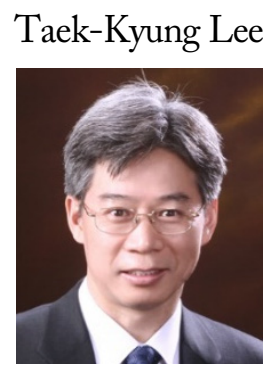

received his B.S. degree in electronic engineering from Korea University in Seoul, Korea in 1983 and his M.S. and Ph.D. degrees in electrical engineering from the Korea Advanced Institute of Science and Technology (KAIST) in 1985 and 1990, respectively. From 1990 to 1991, he served as a postdoctoral fellow at the University of Texas at Austin under a grant from the Korea Science and Engineering Foundation. From August 1991 to February 1992, he worked as a research scientist at KAIST. In March 1992, he joined the faculty of KAU, where he is currently a professor in the School of Electronics and Information Engineering. He was an associate visiting research professor at the University of Illinois at Urbana-Champaign from 2001 to 2002. From 2006 to 2007, he was the chairman of the School of Electronics, Information, and Computer Engineering at KAU, and from 2011 to 2013, he served as the director of the Aerospace and Aviation Electronics Research Center of the same university. Dr. Lee was also the chairman of the Radar Technical Group in the Korea Institute of Electromagnetic Engineering and Science (KIEES) from 2012 to 2013 and president of the institute in 2014. His research interests include computational electromagnetics, antennas, microwave passive circuits, satellite antennas and spaceborne SAR systems, and air surveillance systems.

\section{Sang-burm Ryu}

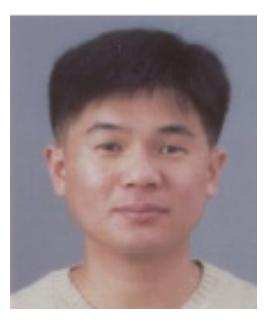

received his B.S. degree in electronic engineering from Daejeon National University of Technology in Daejeon, Korea in 1996 and his M.S. and Ph.D. degrees in the Department of Electronic Engineering from Chungbuk National University, Korea in 2001 and 2010, respectively. Since 2011, he has been working as Senior Engineer with Satellite Payload Development Division, Satellite R\&D Head office,

Korea Aerospace Research Institute, Daejeon, Korea. His research interests include Spaceborne SAR system and satellite communication and Spaceborn Microwave Radiometer.

\section{Hyeon-Cheol Lee}

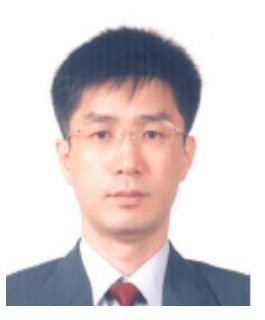

received his B.S. and the M.S. degrees in electronic engineering from Kyungpook National University in 1987 and 1989, respectively, and his Ph.D. degree in electrical engineering from Texas A\&M University in 2001. From 1989 to 1996, he worked at the Agency for Defense Development to repair/operate target tracking control systems that are capable of flying target drones for testing short-range surfaceto-air missile and surveillance unmanned aerial vehicles (UAVs). In 2002, he worked at the Department of Computer Science in Texas A\&M University as a post doctorate, detecting image contours with an adaptive threshold algorithm. Since 2003, he has been working at KARI to develop ultrahigh frequency/Ku-band datalinks and an auto-tracking system for vertical takeoff landing UAV. He has recently began developing satellite SAR payloads.

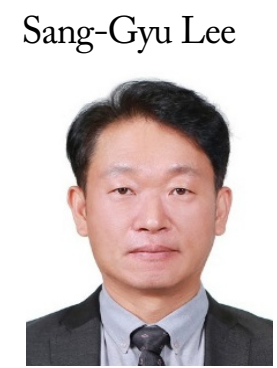

received his B.S., M.S., and Ph.D. degrees in electronic engineering from Chungnam National University in 1993, 1995, and 2011, respectively. During 1993-1995, he focused his research in MPEG2 scalability codec and constant bit rate control mechanism. Since 1995, he has been in charge of development of very high speed satellite image downlink subsystem of KOMPSATs and with the Korea Aerospace Research Institute (KARI), Daejeon, Korea. From 2013 to 2015, he was the program manager of KARI's internal research and development (IRND) program, development of phased array antenna for satellite data transmission. And, since 2014, he has been in charge of the development of radar payload technologies for the compact satellite. He was the head of payload electronics team of KARI from 2015 to 2017. 